\title{
Consequences of late bilingualism for novel word learning: evidence from Tamil- English bilingual speakers
}

Article

Accepted Version

Nair, V. K. K. ORCID: https://orcid.org/0000-0001-6631-0587, Biedermann, B. and Nickels, L. (2016) Consequences of late bilingualism for novel word learning: evidence from TamilEnglish bilingual speakers. International Journal of Bilingualism, 20 (4). pp. 473-487. ISSN 1367-0069 doi: https://doi.org/10.1177/1367006914567005 Available at https://centaur.reading.ac.uk/100974/

It is advisable to refer to the publisher's version if you intend to cite from the work. See Guidance on citing.

Published version at: http://www.scopus.com/inward/record.url?eid=2-s2.0-84982883606\&partnerID=MN8TOARS To link to this article DOI: http://dx.doi.org/10.1177/1367006914567005

Publisher: Sage

All outputs in CentAUR are protected by Intellectual Property Rights law, including copyright law. Copyright and IPR is retained by the creators or other copyright holders. Terms and conditions for use of this material are defined in the End User Agreement. 


\section{CentAUR}

Central Archive at the University of Reading

Reading's research outputs online 
International Journal of Bilingualism O(0)

Article

Consequences of Late Bilingualism for Novel Word Learning: Evidence from Tamil-English Bilingual Speakers

Subtitle: Effects of Late Bilingualism on Novel Word Learning 


\begin{abstract}
Aims and objectives:

Recent studies that have investigated novel word learning have demonstrated an advantage for bilinguals compared to monolinguals (e.g., Kaushanskaya \& Marian, 2009b). The study reported here sought to explore whether a word learning advantage is revealed only for early bilinguals with comparable proficiency in both their languages, or whether such advantages are also observed in individuals with relatively late experience of, and less proficiency in, a second language.
\end{abstract}

\title{
Methodology:
}

We tested the acquisition of novel words in an unknown language using identification and naming tasks in three groups of 20 participants; monolingual Tamil speakers, 'early' TamilEnglish bilingual speakers, and late Tamil-English bilingual speakers.

\section{Data and Analysis:}

The data was analysed using a non-parametric Kruskal-Wallis test followed by linear regressions.

\section{Findings:}

The results showed a bilingual advantage for word learning as evidenced by superior performance in both the naming and identification tasks and, critically, late bilinguals outperformed monolinguals.

\section{Originality:}

The results of the present study revealed, for the first time, a bilingual advantage in word learning even when individuals acquire their second language later in life. 


\section{Significance:}

The results suggest that the positive effects of bilingualism may generalize beyond nonlinguistic tasks, perhaps affecting a general language learning mechanism. Moreover, this seems to occur even in late bilingualism. This is in contrast to the reported effects on cognitive control mechanisms that show only weaker advantages for individuals who learned a second language later in life.

\section{Keywords}

Late bilingualism, novel word learning, late bilingual advantage, phonological memory, socio economic status 


\section{Introduction}

One of the most significant findings to have emerged from research examining bilingualism and cognitive processing is that bilingual children and older adults perform particularly well in tasks involving cognitive processing skills such as selective attention and inhibitory control (e.g., Bialystok, Craik, Klein, \& Viswanathan, 2004; Bialystok, Craik, \& Ryan, 2006; Costa, Hernandez, \& Sebastian-Galles, 2008). Similarly, studies that have investigated the effect of bilingualism on language learning suggest that an early bilingual experience leads to an advantage in novel word learning (e.g., Bartlotti \& Marian, 2012; Bartlotti, Marian, Schroeder, \& Shook, 2011; Grey, 2013; Kaushanskaya, 2012; Kaushanskaya \& Marian, 2009a; Kaushanskaya \& Marian, 2009b, Van Hell \& Mahn, 1997, Wang \& Saffran, 2014; Yoshida, Tran, Bentitez \& Kuwabara, 2011).

Although the advantages of bilingualism for cognitive processing and language learning are well established, the precise nature of the bilingual experience that is needed to obtain a bilingual advantage is not fully understood. For example, the majority of the studies that have reported a bilingual advantage have examined cognitive processing and language learning advantages in early bilinguals with comparable speaking proficiency in both languages. However, bilingual experience can vary in a number of ways. For example, individuals can have varying levels of second language proficiency due to the nature and length of language exposure (high and low proficiency bilinguals). 
They may also have different age of acquisition depending on whether the second language was learned in early or late childhood (early and late bilinguals) and/or distinct patterns of language acquisition (simultaneous and successive bilinguals). Each of these aspects of bilingual experience may interact with bilingual cognitive-linguistic abilities distinctively, yet an understanding of the influence of each of these unique but varied experiences on bilingual cognitive processing or language learning is limited. Although some studies have started to examine the impact of age of second language acquisition on cognitive processing (e.g., Tao, Marzecova, Taft, Asanowicz, \& Wodniecka, 2011), the effects on bilingual language learning abilities are relatively less studied. The purpose of the present study was to examine how the experience of late acquisition of a second language impacts novel word learning ability in bilinguals and to compare their performance with monolinguals and relatively more proficient early bilinguals.

\section{The bilingual advantage in novel word learning}

It has been suggested that a general bilingual advantage for novel word learning is possible in speakers who are early bilinguals. For example, Kaushanskaya and Marian (2009b) examined whether there was a bilingual advantage for novel word learning for early bilinguals, and also investigated how experience with phonologically and orthographically similar or dissimilar languages influenced performance. Previous studies (e.g., Bialystok, Majumder, \& Martin, 2003) had indicated that individuals, who are exposed to languages that are phonologically and orthographically similar (e.g. 
Spanish-English) may show a bilingual advantage in phonological awareness tasks. For example, Bialystok et al. (2003) found a phonological awareness advantage for bilingual children who were exposed to phonologically and orthographically similar languages but that this bilingual advantage was not found for languages with different scripts (e.g. Chinese and English). Kaushanskaya \& Marian (2009b) argued that if exposure to phonologically similar languages can influence phonological awareness, the same effect may be found for novel word learning. They suggested that parallels could be drawn between phonological awareness and novel word learning since both involve phonological processing.

Their adult, early bilingual, participants were native speakers of English and had acquired either Spanish or Mandarin as their second language primarily in a family context. English-Spanish bilinguals (phonologically/orthographically similar languages), English-Mandarin bilinguals (phonologically/orthographically distinct languages) and English monolinguals learned 48 non-words and their English translations. In contrast to predictions, immediate and delayed retention of English translations failed to reveal any learning difference between the two groups of bilinguals. Nevertheless, both groups of bilinguals' demonstrated superior word learning performance compared to monolinguals. While the results have been taken as evidence for a word learning advantage in bilinguals, it is unclear if the translation task used in this study can be taken as a direct measure of word learning. Translation skills 
form an essential component of a bilingual experience (e.g., Malakoff, 1992) therefore; the observed advantage could reflect the better performance of bilinguals on translation tasks rather than any advantage in learning of novel words. Papagno \& Vallar (1995) found a word learning advantage for multilinguals compared to bilinguals, measured using a paired associate word learning task. A number of cognitive-linguistic measures including verbal memory, vocabulary knowledge visuo-spatial span visuo-spatial memory were tested to examine whether differences in phonological memory could account for any word learning advantage demonstrated by multilinguals compared to bilinguals. The results indicated that multilinguals were significantly better at word learning than bilinguals and also showed better auditory digit span and non-word repetition scores, two critical measures of phonological memory. The authors therefore suggested superior phonological memory to be a potential contributor to the better word learning abilities of multilinguals compared to bilinguals. However, correlation does not necessarily mean causation: Though an association between multilingualism, superior word learning and superior phonological memory was evident in the study, it is possible that the multilingual participants already possessed a better phonological memory leading to the acquisition of multiple languages. More recently, Kaushanskaya (2012) provided further evidence of a role for phonological memory in the bilingual word learning advantage. She examined novel word learning in a group of English-Spanish early bilinguals and English monolinguals. Monolingual speakers were divided into 
high and low memory span groups based on their performance on a forward digit span task. The digit span performance of high span monolinguals was equal to that of the bilinguals. Participants were exposed to novel words that were either similar to English phonology (familiar) or dissimilar to English phonology (unfamiliar) and their English translations. Results indicated that bilinguals outperformed both high and low memory span monolinguals and did not show any differences in learning between novel words with familiar phonology and those with unfamiliar phonology. Interestingly, however, depending on their memory span monolinguals exhibited differences in learning of phonologically unfamiliar novel words (but not phonologically familiar novel words): high memory span monolinguals learned phonologically unfamiliar novel words better than low memory span monolinguals. These findings show that, while differences in phonological memory may contribute to novel word learning ability in monolinguals, they cannot fully account for the bilingual advantage in this task. Although this study replicated a bilingual advantage in novel word learning, once again, learning was tested using a translation task which makes it difficult to discern whether the observed advantages were a direct result of differences in learning ability.

In a different set of experiments Kaushanskaya \& Rechtzigel (2012) examined whether the bilingual advantage in novel word learning varied depending on whether words were concrete or abstract. Their manipulation was based on previous evidence suggesting a learning advantage for concrete words than abstract words due their robust lexical- 
semantic representations (e.g., De Groot \& Keijzer, 2000). They suggested that the effect of concreteness might be larger for bilinguals for two reasons a) the bilingual ability to learn novel words may be partially rooted in their ability to better encode semantic information. This might lead to an advantage in learning words that contain rich semantic information; b) concrete words might activate semantic information in both languages for bilinguals compared to monolinguals. The authors predicted that this wider activation of semantic information would therefore facilitate acquisition of concrete words more than abstract words. English-Spanish late bilinguals and English monolinguals learned concrete and abstract novel words through a translation task. Reaction time data failed to show any significant difference in performance between two groups however accuracy data revealed an overall benefit of concreteness for both bilinguals and monolinguals. Furthermore bilinguals showed a larger (almost double) effect of concreteness compared to monolinguals, suggesting a greater bilingual sensitivity to semantic information during novel word learning.

Kaushanskaya, Yoo, \& Van Hecke (2013) investigated whether acquisition of novel words in bilinguals was constrained by the phonological properties of novel words. The authors also examined how degree of second language experience interacted with novel word learning. Native speakers of English with varying level of Spanish second language knowledge (experienced and inexperienced) were tested using a forced choice recognition task for the acquisition of phonologically familiar and unfamiliar novel 
words paired with either a known (animal) or unknown referent (alien). Both groups of participants showed an effect of phonological familiarity that was significant only when the novel words were paired with a familiar referent. Experienced second language learners also only outperformed inexperienced learner, under the same conditions (when unfamiliar novel words were paired with a familiar referent). These findings indicate that increased second language experience affects word learning however such effects in bilinguals may be modulated by whether the referent with which the novel word is paired already has a word referent in the participants vocabulary (ie animals vs aliens). It has also been shown that a bilingual advantage in novel word learning varies relative to the task demands. For example, Kan \& Sadagopan (2014) found no difference in the performance between monolingual and bilingual young adults on novel word retention abilities when measured through either comprehension or naming probes. However a previous study conducted by Kan, Sadagopan, Janich \& Andrade (2013) did find a specific bilingual advantage for comprehension scores, although still not for word production.

Evidence also suggests a distinct neural activation pattern for word learning in bilinguals and monolinguals. For example, Bradley, King \& Hernandez (2013) gave novels words in German to Spanish-English bilinguals and English monolinguals to identify whether cognitive control mechanism responsible for novel word learning in bilinguals and monolinguals differed. Although their word accuracy did not suggest 
any significant difference between monolinguals and bilinguals the reactions time data indicated an overall faster reaction time for bilinguals. Critically, the neuroimaging data revealed that the bilingual brain activation was constrained to specific regions (e.g., putamen). In contrast, the results indicated that monolinguals had a wider activation of brain regions. The authors concluded that a wider activation of brain regions is associated with slower reaction times and this may explain the increased monolingual difficulties in retrieving learned novel words during learning.

\section{Bilingual advantage in late bilinguals}

To the best of our knowledge, there is only one study that has explicitly probed the effect of age of acquisition of a second language on novel word learning in bilinguals: Kaushanskaya \& Marian (2007) compared monolinguals with high proficiency early bilinguals (second language acquisition around 3 years of age) and high proficiency late bilinguals (around 12 years). Participants heard 48 non-words and saw their English translations. During production and identification probes, participants heard a nonword, and were required to produce the associated English translations and then selected the correct English translation from a choice of five. Superior learning performance was observed in early bilinguals compared to monolinguals indicating a bilingual advantage for novel word learning. However, despite having comparable second language proficiency to the early bilinguals, the late bilinguals did not demonstrate a significant 
advantage over monolinguals. This prompted the authors to suggest that the bilingual advantage in novel word learning was sensitive to age of acquisition.

\section{The present study}

The findings from a small number of studies in the literature confirm a bilingual advantage for word learning. However, it is not clear whether such an advantage is only seen for individuals with extensive knowledge of many languages (Papagno \& Vallar, 1995) or in cases of early bilinguals (Kaushanskaya, 2012; Kaushanskaya \& Marian, 2009b). The effect of bilingualism on novel learning has been insufficiently investigated, with the exception being Kaushanskaya \& Marian (2007), and much remains to be explored, particularly regarding novel word learning in late bilingual individuals with limited second language proficiency.

The present study focuses on the impact of late language experience on novel word learning. One of the key learning demands for a late second language learner is to master many novel words. Therefore, it is possible that this experience positively affects the word learning mechanism leading to a bilingual advantage. Alternatively, it is possible that for late bilinguals, the language learning system is relatively less amenable to positive effects of additional experience (unlike that of early bilinguals) resulting in no bilingual advantage. To investigate these possibilities, we examined novel word learning performance in late bilinguals with low second language proficiency and 
compared their performance to monolinguals and proficient bilinguals who acquired their second language earlier.

In contrast to the majority of previous studies, which have used translation tasks, (Kaushanskaya \& Marian, 2007; Kaushanskaya \& Marian, 2009b, Kaushanskaya 2012) we used a novel word learning task which probed learning through a picture naming and identification task. If bilingual experience results in a novel word learning advantage, we predicted that even a late bilingual experience may affect the language learning mechanism which may then facilitate foreign vocabulary acquisition. This predicts that late bilinguals should perform better than monolinguals and possibly even comparably to early bilinguals in learning.

\section{Method}

\section{Participants}

The participants were 60 adults in three age-matched groups: 20 Tamil monolinguals and 40 Tamil-English bilinguals in two groups of 20 (see below for further details). Background data for the participant groups are given in Table 1. The monolinguals (11 males and 9 females) received their education from the local government schools that promoted native language literacy instruction. None of the monolingual participants reported any exposure to English or to any other language at home or in their work environment. 
The bilingual participants rated their language competence across four language modalities (speaking, understanding, reading, writing) on a scale ranging from 0 (not proficient) to 4 (highly proficient) based on the Chengappa, Shivashankar, Nair, Nayak \& Arvind (2011) test of language proficiency. The bilingual participants were divided into two groups (Table 1) based on their age of second language (L2) acquisition for speaking and language proficiency: One group we label 'early' bilinguals (with high proficiency) and the second 'late' bilinguals (with low proficiency). The mean age of onset of speaking in L2 was the primary measure as it generally indicates the start of active bilingualism (Luk, De Sa, \& Bialysok, 2011).

The 'early' bilinguals (13 males and 7 females) were native speakers of Tamil (L1) and acquired English (L2) as a second language. Sixteen of these participants reported that their initial exposure to L2 was in a formal and educational setting and the remaining four participants were exposed to L2 (reading and writing) informally in a family context prior to their schooling. For all 'early' bilingual participants, their schooling was entirely in English (high exposure). Our definition of 'early' bilinguals was based on being exposed to L2 by the age of 5 (earlier than late bilinguals), onset of fluent speaking by 9 years and being immersed in an English speaking environment during schooling. For our 'early' bilingual participants the mean onset age of L2 introduction (for reading and writing) was 4.7 years $(\mathrm{SD}=0.46$; maximum age $=5.0$ ) and for fluent speaking was 8.3years $(\mathrm{SD}=0.44$, maximum age $=9.0)$. 
Late bilinguals (11 males and 9 females) had a delayed onset of introduction to L2. L2 was introduced to them as a restricted part of the school curriculum in an English language lesson primarily through reading and writing (mean onset age $=6.2$, $\mathrm{SD}=0.84 ;$ minimum age $=6.0)$ with a mean onset age for fluent speaking of 12.45 years $(\mathrm{SD}=0.73$, minimum age $=12.0)$. The late bilinguals reported that the delayed onset age for speaking in their L2 had resulted in lesser competence in L2 compared to L1. The late bilinguals were disadvantaged as their exposure to L2 was restricted (low exposure) and therefore L2 acquisition was slow and limited. Late bilinguals and 'early' bilinguals differed significantly in both age of onset of bilingualism measured by speaking and rated proficiency in all language modalities (see Table 1). Although our 'early' bilinguals became fluent in their L2 four years earlier than our late bilinguals, they still acquired their L2 relatively late. Nevertheless, we wished to examine whether even with this, perhaps weak, division, there would be a significant difference between the groups in their word learning.

Linguistic context of the participants. The bilingual and monolingual participants were all from Coimbatore, a town located in Tamil Nadu, a Southern Indian state. Tamil is the most widely used spoken language by the local population. English is mainly taught in schools as a second language to meet educational needs. Although Hindi is one of the 22 official languages of the Indian subcontinent, it is not used as a medium of 
communication in major parts of Southern India. In Tamil Nadu, Hindi is extremely unpopular, and rarely encountered, as a medium of communication or as a language of popular culture for historic and political reasons (see Forrester, 1966; Pandian, 1996 for detail on this topic). The participants in the current study reported no prior exposure to this language.

Participant demographics and background measures. The participants completed a non-word repetition task as a measure of phonological short-term memory. Previous studies have indicated that phonological short-term memory can influence word learning (e.g., Gupta, 2003). Non-word repetition is one of the most widely used methods to assess phonological short-term memory. In this task, participants were presented with ten non-words within the range of 2-8 syllables that had the phonology of Tamil. Each non-word was presented separately with increasing syllable length via headphones. The participant's task was to repeat the single presented non-word. A score of 1 was given for each correct response with no production errors.

As shown in Table 1, univariate ANOVA showed a significant effect of group on nonword repetition performance $\left[\mathrm{F}(2,38)=3.67, \mathrm{p}=.035, \eta^{2}=.22\right]$. Bonferroni adjusted significance tests for pairwise comparisons revealed that monolinguals showed significantly poorer nonword repetition compared to 'early' bilinguals (mean difference $=-.75,95 \% \mathrm{CI}:[-1.45,-.04], \mathrm{p}=.034)$. However, there were no significant differences 
between late bilinguals and either monolinguals (mean difference $=-.25,95 \%$ CI: $[-.45$, $.95], \mathrm{p}=.252$ ) or 'early' bilinguals (mean difference $=-.50,95 \%$ CI: $[-1.20, .20], \mathrm{p}=$ .252). Socio-economic status (SES) of the participants was assessed based on four critical indicators (pooled monthly income, highest education, and occupation and family properties) by socio economic status scale (Venkatesan, 2011). There was a significant difference in SES between all three groups: $[\mathrm{F}(2,57)=298.57, \mathrm{p}<.001$, $\left.\eta^{2}=.91\right]$. Bonferroni adjusted significance tests for pairwise comparisons revealed significantly lower SES scores for monolinguals compared to 'early' bilinguals (mean difference $=-4.25,95 \% \mathrm{CI}:[-5.175,-3.32], \mathrm{p}<.001)$ and late bilinguals [mean difference $=-4 ., 95 \%$ CI: $[-5.87,-4.02, p<.001]$. Late bilinguals also showed significantly lower SES scores compared to 'early' bilinguals (mean difference $=9.20$, 95\% CI: $[-8.27,10.12, \mathrm{p}<.001]$. The implications of these results are discussed further below. 
Table 1. Demographic and background data of participants as means and standard deviations (in parentheses).

\begin{tabular}{|c|c|c|c|c|}
\hline Demographic data & Mono & LateBi & EarlyBi & $\mathbf{p}$ \\
\hline Age (years) & $22.7(1.45)$ & $22.1(2.02)$ & $21.6(1.68)$ & .328 \\
\hline Socio economic status ${ }^{\mathrm{a}}$ & $5.04(0.21)$ & $9.28(0.82)$ & $14.23(1.77)$ & $<.001$ \\
\hline Non-word repetition ${ }^{\mathrm{b}}(\mathrm{n}=10)$ & $6.80(0.81)$ & $7.05(.66)$ & $7.55(1.02)$ & .035 \\
\hline L2 acquisition age (speaking) & - & $12.45(0.73)$ & $8.3(0.44)$ & $<.001$ \\
\hline \multicolumn{5}{|l|}{ Proficiency ratings $^{c}$} \\
\hline Speaking & - & $2.42(0.49)$ & $3.15(0.36)$ & $<.001$ \\
\hline Listening & - & $2.57(0.49)$ & $3.68(0.46)$ & $<.001$ \\
\hline Reading & - & $2.52(0.51)$ & $3.55(0.51)$ & $<.001$ \\
\hline Writing & - & $1.93(0.30)$ & $3.42(0.50)$ & $<001$ \\
\hline \multicolumn{5}{|c|}{$\begin{array}{l}\text { Notes } \\
\mathrm{N}=20 \text { for all three participant groups } \\
\text { Mono:Monolinguals, LateBi:Late Bilinguals, EarlyBi:'Early' Bilinguals } \\
\mathrm{p}=\text { significance of univariate ANOVA between three language groups for age, nonword repetition and SES } \\
\text { and significance of t-test ( } 2 \text { tailed) for proficiency ratings between late and 'early' bilinguals. } \\
\text { a'Scale from } 0 \text { (lowest SES) to } 20 \text { (highest SES). Pairwise group performance for SES are reported above } \\
{ }^{b} \text { The pairwise group performance for non-word repetition are reported above. } \\
\text { chatings from } 0=\text { not proficient to } 4=\text { highly proficient }\end{array}$} \\
\hline
\end{tabular}




\section{Stimuli}

The novel word stimuli and pictures (see Appendix 1) were selected from the Hindi picture word articulation test (Kacker, Basavaraj, Thapar, Menon \& Vasudeva, 1990). The test consists of 68 coloured photographs of objects with disyllabic Hindi names for assessing the production of 48 speech sounds in the initial, medial and final position. For the purpose of the present study, we selected ten target words as learning stimuli. The target words selected were all common nouns with no close word similarity to Tamil or English. The ratings for word similarity for both the languages were carried out by 5 Tamil-English bilingual speech pathologists based on a 1-4 point rating scale $(1=$ no resemblance with Tamil or English, $4=$ close resemblance with Tamil or English). Ratings indicated that the novel words did not show any close resemblance to either Tamil $(\mathrm{M}=1.06, \mathrm{SD}=0.25)$ or English $(\mathrm{M}=1.13, \mathrm{SD}=0.32)$. The novel words did not contain any non-native phonemes of Tamil. Each novel word was paired with a picture corresponding to its Hindi referent. The learning stimuli were audio recorded by a fluent speaker of Hindi. Audio files of all stimuli were recorded by a native Hindi speaker and presented in combination with the pictures using Powerpoint.

\section{Procedure}

Before the familiarisation and learning phase began, the participants filled in a language proficiency questionnaire and performed the background testing. The participants then 
sat in front of a computer (Compaq Presario V6425TU laptop) in a soundproof room. The entire session (background, familiarisation, learning and test) lasted for two hours.

Familiarisation phase. The session started with a familiarisation phase, where participants were each presented with each target word and its corresponding picture once. The familiarisation phase included an introduction to the learning session (see instructions below), learning phase and an opportunity to ask questions. In the introduction they were instructed: "You will hear some words from another language that is unknown to you. You must pay keen attention to these words and their corresponding pictures. It is important to listen to all the words correctly because your ability to remember these words will be tested immediately after the learning."

The participants were told about the entire task and were made aware that repetition of a word or an entire learning session was not possible. After the introduction participants were asked to carefully listen to each novel word presented auditorily through headphones and simultaneously look at the picture of the referent that was displayed on the computer monitor.

At the end of familiarisation phase, participants were encouraged to ask questions regarding the task and were provided with answers before the start of the learning phase. The approximate duration of this phase was 20-30 minutes. 
Learning phase. In the learning phase, all the novel word stimuli were presented repeatedly three times in random order. The stimuli were randomised individually for each participant. Each presentation consisted of hearing the auditory stimulus and seeing its visual referent. The word and picture appeared simultaneously and the picture remained on the computer screen for around 30 seconds. The duration of picture presentation was constant across participants for all learning sessions. The participants sat silently during stimulus presentation. At the end of the third presentation, in order to enhance the effect of learning, participants performed a repetition phase: after listening to each target word on headphones they repeated the novel words aloud three times (no pictures were presented). The approximate duration of each learning session was 5 minutes, with the final session taking approximately 8-10 minutes due to the repetition component.

Test phase. At the end of the learning phase, the acquisition of these novel words was assessed using picture naming and identification tasks.

In the picture naming task, the participant was asked to name the picture of the target referent using the newly learned Hindi word. Responses were audio-recorded and phonetically transcribed using the International Phonetic Alphabet (IPA) for later analysis.

In the identification task, a set of three picture choices were provided. The picture choices included the target referent and two semantically unrelated distractors 
from the stimulus set (none of the words were repeated more than 3 times). The target word was then presented auditorily and the participant was instructed to point to the picture that corresponded to the auditory stimulus. For both naming and identification a maximum response time of one minute was provided. However, if the responses were obtained before one minute then the next target word was presented. Testing lasted for approximately $15-20$ minutes.

\section{Analysis}

Responses were analysed based on the identification and naming accuracy scores for all the three groups (monolinguals, late bilinguals and 'early' bilinguals). Naming responses were classified as correct when the participant produced the novel word with $100 \%$ accuracy. Words with production errors (e.g., omission, repetition, substitution of phonemes, naming a wrong word from the target items) were considered as incorrect responses. 


\section{Results}

Participants' naming and identification accuracy are given in Figures 1 and 2. For both tasks, as there was a ceiling effect in the data (for 'early' bilinguals) we therefore used a non-parametric Kruskal-Wallis test to examine the effects of group (monolingual, late bilingual, 'early' bilingual) on performance followed by planned pairwise comparisons.

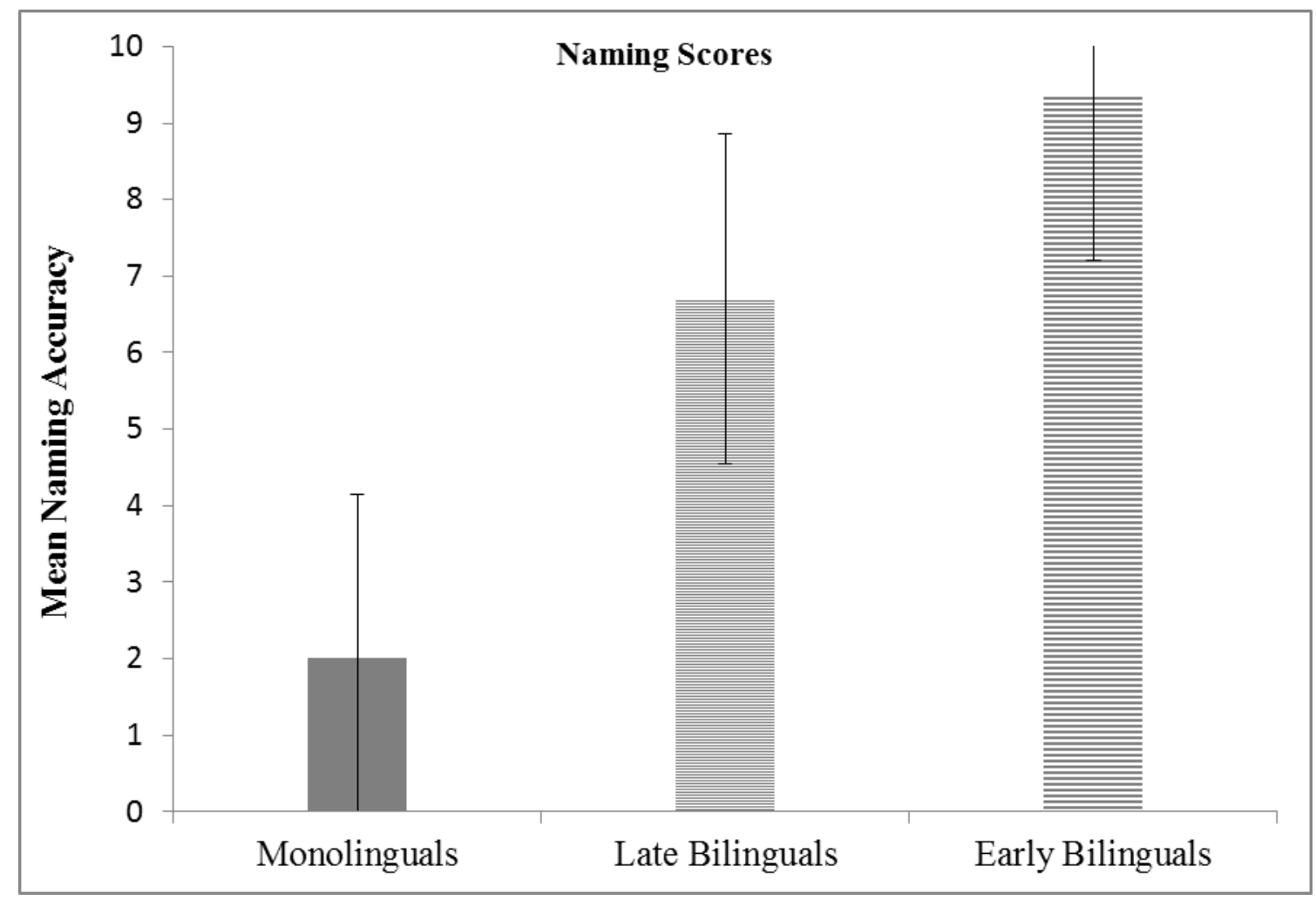

Figure 1. Participants' mean naming accuracy (maximum score $=10$ ), Error bars = Standard Error. 
There was a significant difference in naming accuracy across the groups (Kruskal Wallis: $\left.\chi^{2}(2)=49.38, p<.001\right)$. Bonferroni adjusted significance tests for pairwise comparisons revealed a significant difference for naming scores between all the groups (monolinguals $($ mean rank $=10.55)$ and late bilinguals $($ mean rank $=32.00), \mathrm{p}<.001$, monolinguals (mean rank $=10.55)$ and 'early' bilinguals (mean rank $=48.95), \mathrm{p}<.001$ and between late (mean rank $=32.00$ ) and 'early' bilinguals (mean rank $=48.95), p<$ $.001)$.

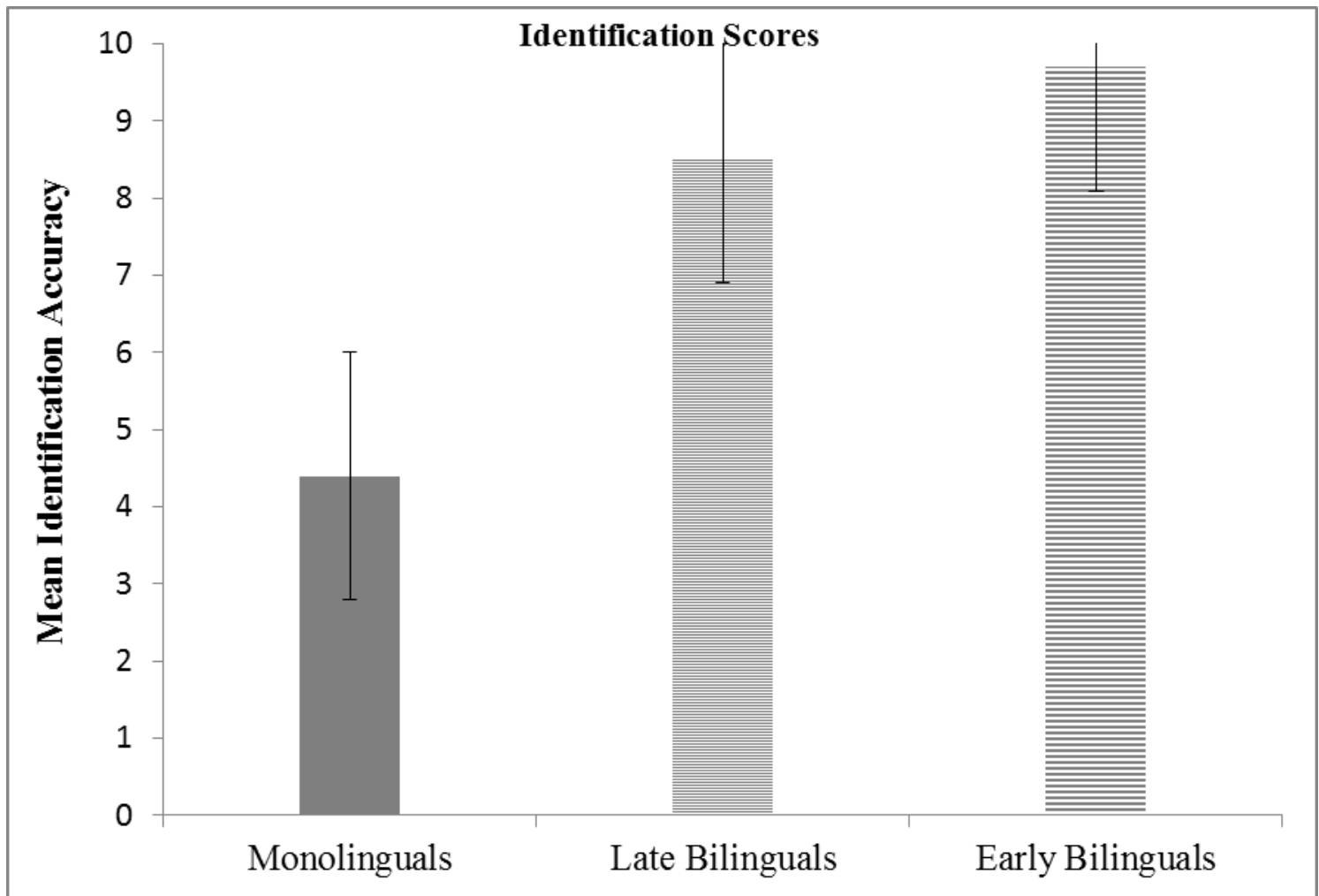


Figure 2. Participants' mean identification accuracy (maximum score $=10$ ), Error bars = Standard Error.

A significant overall main group effect was also evident for identification scores, $\chi^{2}(2)$ $=44.34, \mathrm{p}<.001$. Bonferroni adjusted significance tests for pairwise comparisons revealed a significant difference for identification scores between monolinguals and both the bilingual groups (monolinguals (mean rank $=10.98$ ) and late bilinguals (mean rank $=34.93), \mathrm{p}<.001$, monolinguals (mean rank = 10.98) and 'early' bilinguals (mean rank $=45.60), \mathrm{p}<.001)$. However, there was no significant difference between late $($ mean rank $=34.93)$ and 'early' bilinguals (mean rank $=45.60)(\mathrm{p}=.135)$.

Controlling background differences using Analysis of covariance (ANCOVA)

The results clearly showed a gradient of performance across groups, with both bilingual groups performing better than the monolingual group, and the 'early' bilinguals outperforming the late bilinguals (at least for naming). However, our background data revealed that there were significant differences in both SES and phonological memory (indexed by nonword repetition). Consequently, we also carried out an ANCOVA collapsing the word learning scores of two bilingual groups into a single group to eliminate the ceiling effect and comparing this group with the monolingual group while controlling for SES and nonword repetition. The effect of group on performance remained significant for both naming $\left[F(1,56)=31.35, p<.001, \eta^{2}=.359\right]$ and 
identification tasks $\left[\mathrm{F}(1,56)=42.02, \mathrm{p}<.001, \eta^{2}=.429\right]$, indicating that it was bilingualism rather than SES or phonological short-term memory that influenced novel word learning.

Exploring the effects of age of onset of bilingualism: Regression analysis

Our final analysis examined in more detail the effect of age of onset of bilingualism (indexed by speaking) on word learning, while controlling for SES and phonological memory (Pearson's correlations were carried out prior to these regressions and are reported in Appendix 2). This regression confirmed that age of onset of bilingualism is a statistically significant predictor of word learning, even when SES and phonological memory are controlled for (see Table 2).

Table 2. Regression results examining predictors of word learning in bilinguals.

$\begin{array}{llllllll}\text { DepdVari } & \text { IndVari } & \text { B } & \text { SE } & \beta & \text { p value } & \text { R Square } & \text { OMS }\end{array}$

Naming

$.489 \quad \mathrm{p}<.01$

$\begin{array}{lllll}\mathrm{AOB}(\mathrm{S}) & -.571 & .179 & -.722 & .003 \\ \mathrm{SES} & -.002 & .147 & -.003 & .989 \\ \mathrm{NWR} & .212 & .253 & .104 & .409\end{array}$

Identification

$.307 \quad .004$

$\mathrm{AOB}(\mathrm{S}) \quad-.445 \quad .146 \quad-.801 \quad .004$


$\begin{array}{lllll}\text { SES } \quad-.138 & .120 & -.307 \quad .258\end{array}$

$\begin{array}{lllll}\text { NWR } & .217 & .207 & .152 & .303\end{array}$

Note DepdVari $=$ Dependent variable, IndVari $=$ Independent Variable, $\mathrm{SE}=$ Standard Error, $\mathrm{R}$ Square $=\mathrm{R}$ square (adjusted), $\mathrm{OMS}=$ Overall model significance, $\mathrm{AOB}(\mathrm{S})=$ age of onset of bilingualism indexed by speaking.

\section{Discussion}

In order to investigate whether the language learning mechanism is affected by bilingual experience, we examined the acquisition of novel words in monolinguals, late bilinguals and 'early' bilinguals. As predicted from previous research, we found a clear effect of bilingualism with 'early' bilinguals showing superior word learning compared to monolinguals (Kaushanskaya \& Marian, 2009b; Kaushanskaya, 2012). However, critically, even the bilingual group with a later onset of bilingualism (the late bilingual group) also outperformed monolinguals and demonstrated a bilingual advantage in novel word learning, despite low proficiency and relatively late age of onset of bilingualism. This indicates that even when delayed, exposure to another language exerts a significant influence on language learning for bilingual speakers and facilitates word learning skills. The late bilingual advantage observed in the present study is in 
contrast with previous findings that only 'early' bilinguals benefitted from their bilingualism in novel word learning (Kaushanskaya \& Marian, 2007). Indeed, even our 'early' bilinguals had acquired their second language relatively late, and later than the early bilinguals in other studies. Nevertheless, late bilinguals, despite showing an advantage over monolinguals, showed significantly poorer word learning than 'early' bilinguals, and overall age of onset of bilingualism was a significant predictor of word learning ability. This could be an effect of either proficiency or length of exposure, as these two were confounded in our sample (and indeed often will be). This replicates the earlier findings by Kaushanskaya \& Marian (2007) who found an effect of length of exposure within late bilinguals on word learning measured through translation. That is, late bilinguals with longer second language exposure outperformed their counterparts with shorter exposure. Further research is required to determine whether length of exposure is a critical variable influencing the extent of a bilingual advantage in word learning.

Recently there have been proposals to consider bilingual word learning within the framework of bilingual inhibitory control (Kaushanskaya \& Marian, 2009b; Kaushanskaya, 2012). The general idea is that tasks examining word learning skills involve selection of a target word and successful inhibition of all irrelevant words that are activated during target word learning (Kaushanskaya \& Marian, 2009b). A robust bilingual advantage in inhibitory control has been often suggested for early bilinguals 
compared to late bilinguals (Tao et al., 2011). Therefore the performance difference between early and late bilinguals may be due to the differences in inhibitory control abilities. Inhibitory control is particularly required when the referents are familiar (the referent familiarity effect: Kaushanskaya, Yoo, \& Van Hecke, 2013) as, for these stimuli, a word already exists in the participants' other language(s) and this word will have to be inhibited.

However, the critical question is why did we observe an advantage in novel word learning for late bilinguals compared to monolinguals? Novel word learning involves encoding of unfamiliar phonological information (Kaushanskaya \& Marian, 2009b) and mapping the phonological form onto its respective semantic referent. For the second language learner, there is a great emphasis on acquiring novel words. This leads to significantly increased experience in encoding unfamiliar phonological information.

This experience may result in greater proficiency in novel phonological encoding leading to an advantage for novel word learning in late bilinguals. It is possible that late bilinguals may only show an advantage in tasks that resemble learning conditions similar to second language acquisition (e.g., picture naming task in a classroom context). Once again, when, as in our experiment, familiar referents are used and therefore there is a need for inhibition of the name of the referent in the native language. This situation is commonplace for bilinguals (even later bilinguals), but for monolinguals is more rarely encountered. Hence, the advantage, even for late bilinguals, 
once again could be related to inhibitory control required for learning and retrieving new names for familiar referents.

A main difference from previous studies is that we tested learning using naming and identification rather than a translation task. This required all participants to retrieve novel words when presented with pictures rather than from a word in their first language. Although this confirms that an advantage in novel word learning is not a mere reflection of bilinguals' translation ability, it is likely that factors that are associated with task type would have influenced the learning. For instance, recent findings by Kan, Sadagopan, Janich \& Andrade (2013) can be taken as an evidence for such task effects where the bilinguals showed a specific advantage for comprehension probes when word learning was assessed through a fast mapping task. Although in our study the 'early' bilinguals performed well, the learning task remained challenging for late bilinguals and particularly monolinguals, despite the fact that our study used only ten novel words for learning. This is considerably fewer than other studies (e.g. Kaushanskaya \& Marian, 2009b; Kaushanskaya, 2012) and suggests that perhaps translation tasks are easier than naming. It is also possible that combination of the nature of the task and the relatively small number of items interacted to facilitate word learning specifically for our 'early' bilinguals.

Two other major factors should be considered in relation to our results: phonological short-term memory and SES. Phonological short-term memory has long 
been established as a strong predictor in the acquisition of novel words (e.g., Baddley, Gathercole, \& Papagno, 2008). Papagno \& Vallar (1995) have previously argued that a superior phonological short-term memory in multilinguals may enhance foreign vocabulary learning. There was indeed a significant difference across our groups in phonological short-term memory (measured using a non-word repetition task), and nonword repetition scores were predictors of naming and identification. Although this results indicates an effect of phonological memory on novel word learning bilingual status remained a predictor over and above these effects. This finding supports other reports (e.g., Kaushanskaya, 2012) that the differences in bilingual word learning needs to be accounted through measures beyond differences in phonological memory performance.

The three groups of participants in the present study also differed in their SES. The monolinguals came from a lower socio economic status than the late bilinguals who in turn were of lower SES than the 'early' bilinguals. This is quite different from an American context where early bilingualism is often linked with lower SES (Costa \& Sebastián-Gallés, 2014). The role of higher SES in facilitating language and neurocognitive performance has been established in children (Noble, Norman \& Farah, 2005). It has also been found that SES and bilingualism may influence language (vocabulary) and executive functioning abilities independently (Calvo \& Bialystok, 2014). While the relationship between adult word learning and SES remains unclear and 
worthy of future investigation, given that fact that SES is positively correlated with vocabulary acquisition, it may be reasonable to assume that higher SES could be a potential contributor towards an enhanced word learning skills in 'early' bilingual performance. However, while SES was correlated with word learning score, once bilingual status and phonological working memory was taken into account in the regression, there was no longer a significant effect on word learning. This finding is in line with reports from cognitive processing literature suggesting that a bilingual advantage in cognitive control is not confounded by SES (de Abreu, Cruz-Santos, Tourinho, Martin \& Bialystok, 2012).

In conclusion, bilinguals in our study were more adept at learning novel words than monolinguals and this could not be accounted by differences in SES or phonological short term memory. These results indicate (i) unlike the cognitive advantage, even late bilingualism contributes a novel word learning advantage; and (ii) there is a direct association between extent of second language experience and word learning. We suggest that the late bilingual advantage in word learning may be restricted to only word learning tasks that bear significant similarities to vocabulary acquisition in second language bilingual word learning environments. Further research is required to test this hypothesis. Similarly, investigating the effect of other factors such as inhibitory control (Kaushanskaya, 2012) and SES in late bilingual word learning will offer further insights into the mechanisms underlying such advantages. 


\section{References}

Baddeley, A., Gathercole, S., \& Papagno, C. (1998). The phonological loop as a language learning device. Psychological review, 105(1), 158.

Bartolotti, J., \& Marian, V. (2012). Language learning and control in monolinguals and bilinguals. Cognitive science, 36(6), 1129-1147.

Bartolotti, J., Marian, V., Schroeder, S. R., \& Shook, A. (2011). Bilingualism and inhibitory control influence statistical learning of novel word forms. Frontiers in psychology, 2: 324 .

Bialystok, E., Majumder, S., \& Martin, M. M. (2003). Developing phonological awareness: Is there a bilingual advantage?. Applied Psycholinguistics, 24(01), 27-44.

Bialystok, E., Craik, F. I., Klein, R., \& Viswanathan, M. (2004). Bilingualism, aging, and cognitive control: evidence from the Simon task. Psychology and aging, 19(2), 290.

Bialystok, E., Craik, F. I., \& Ryan, J. (2006). Executive control in a modified antisaccade task: Effects of aging and bilingualism. Journal of Experimental Psychology: Learning, Memory, and Cognition, 32(6), 1341. 
Bradley, K. A., King, K. E., \& Hernandez, A. E. (2013). Language experience differentiates prefrontal and subcortical activation of the cognitive control network in novel word learning. NeuroImage, 67, 101-110.

Calvo, A., \& Bialystok, E. (2014). Independent effects of bilingualism and socioeconomic status on language ability and executive functioning. Cognition, 130(3), 278-288.

Chengappa, S., Shivashankar, N., Nair, K. K Vishnu., Nayak, D \& Arvind, H. (2011). Test of Language Proficiency. Language and Brain Organization in Normative Multilingualism. Department of Science and Technology (DST), Government of India.

Costa, A., Hernández, M., \& Sebastián-Gallés, N. (2008). Bilingualism aids conflict resolution: Evidence from the ANT task. Cognition, 106(1), 59-86.

Costa, A., \& Sebastian-Galles, N. (2014). How does the bilingual experience sculpt the brian. Nature Reviews Neuroscience. 15, 336-345.

de Abreu, P. M. E., Cruz-Santos, A., Tourinho, C. J., Martin, R., \& Bialystok, E. (2012). Bilingualism enriches the poor enhanced cognitive control in low-income minority children. Psychological science, 23(11), 1364-1371. 
De Groot, A., \& Keijzer, R. (2000). What is hard to learn is easy to forget: The roles of word concreteness, cognate status, and word frequency in foreign-language vocabulary learning and forgetting. Language Learning, 50(1), 1-56.

Forrester, D. B. (1966). The Madras anti-Hindi agitation, 1965: Political protest and its effects on language policy in India. Pacific Affairs, 19-36.

Grey, S. E. (2013). A neurocognitive investigation of bilingual advantages at additional language learning. Georgetown University.

Gupta, P. (2003). Examining the relationship between word learning, nonword repetition, and immediate serial recall in adults. The Quarterly Journal of Experimental Psychology: Section A, 56(7), 1213-1236.

Kacker, S.K., Basavaraj, V., Thapar, A., Menon, N., \& Vasudeva, R. (1990). Hindi Picture Word Articulation Test. SAFA Project. AIIMS, New Delhi.

Kan, P. F., Sadagopan, N., Janich, L., \& Andrade, M. (2013). Effects of Speech Practice on Fast Mapping in Monolingual and Bilingual Speakers. Journal of Speech, Language, and Hearing Research, 1-13.

Kan, P. F., \& Sadagopan, N. (2014). Novel word retention in bilingual and monolingual speakers. Language Sciences, 5, 1024. 
Kaushanskaya, M. (2012). Cognitive mechanisms of word learning in bilingual and monolingual adults: The role of phonological memory. Bilingualism: Language and Cognition, 15(03), 470-489.

Kaushanskaya, M., \& Marian, V. (2008). Age-of-Acquisition Effects in the Development of a Bilingual Advantage for Word Learning. In Proceedings of the 32nd Annual Boston University Conference on Language Development (pp. 213-224).

Kaushanskaya, M., \& Marian, V. (2009a). Bilingualism reduces native-language interference during novel word learning. Journal of Experimental Psychology: Learning, Memory, and Cognition, 35(3), 829.

Kaushanskaya, M., \& Marian, V. (2009b). The bilingual advantage in novel word learning. Psychonomic Bulletin \& Review, 16(4), 705-710.

Kaushanskaya, M., \& Rechtzigel, K. (2012). Concreteness effects in bilingual and monolingual word learning. Psychonomic bulletin \& review, 19(5), 935-941.

Kaushanskaya, M., Yoo, J., \& Van Hecke, S. (2013). Word Learning in Adults With Second-Language Experience: Effects of Phonological and Referent Familiarity. Journal of Speech, Language, and Hearing Research, 56(2), 667-678. 
Luk, G., De Sa, E., \& Bialystok, E. (2011). Is there a relation between onset age of bilingualism and enhancement of cognitive control?. Bilingualism: Language and Cognition, 14(04), 588-595.

Malakoff, M. E. (1992). Translation ability: A natural bilingual and metalinguistic skill. Advances in psychology, 83, 515-529.

Noble, K. G., Norman, M. F., \& Farah, M. J. (2005). Neurocognitive correlates of socioeconomic status in kindergarten children. Developmental science, 8(1), 74-87.

Papagno, C., \& Vallar, G. (1995). Verbal short-term memory and vocabulary learning in polyglots. The Quarterly Journal of Experimental Psychology, 48(1), 98-107.

Pandian, M. S. S. (1996). Towards National-Popular: Notes on Self-Respecters' Tamil. Economic and political weekly, 3323-3329.

Tao, L., Marzecová, A., Taft, M., Asanowicz, D., \& Wodniecka, Z. (2011). The efficiency of attentional networks in early and late bilinguals: The role of age of acquisition. Frontiers in Psychology, 2: 123.

Van Hell, J. G., \& Mahn, A. C. (1997). Keyword mnemonics versus rote rehearsal: Learning concrete and abstract foreign words by experienced and inexperienced learners. Language Learning, 47(3), 507-546. 
Venkatesan, S. (2011). Socio Economic Status Scale: NIMH revised version. AIISH, Mysore.

Wang, T., \& Saffran, J. R. (2014). Statistical learning of a tonal language: the influence of bilingualism and previous linguistic experience. Frontiers in psychology, 5:953.

Yoshida, H., Tran, D. N., Benitez, V., \& Kuwabara, M. (2011). Inhibition and adjective learning in bilingual and monolingual children. Developmental Psychology, 2, 210. 


\section{Appendix 1}

Novel words and English translations

\begin{tabular}{|c|c|c|}
\hline Novel words & IPA & Translations in English \\
\hline Hathi & ha:th $t_{n}^{h}$ & Elephant \\
\hline Chata & t $\int^{\text {ha:ta: }}$ & Umbrella \\
\hline Ainak & ænək & Spectacles \\
\hline Kaechi & kæ̃t $\int \mathrm{I}$ & Scissors \\
\hline Chammach & t fammots & Spoon \\
\hline Batak & batak & Duck \\
\hline Kitab & kita:b & Book \\
\hline Patang & patan & Flag \\
\hline Chuha & t fu:ha & Rat \\
\hline Kutha & kutta & Dog \\
\hline
\end{tabular}




\section{Appendix 2}

Correlation analysis with age of onset of bilingualism as measured by speaking $(\mathrm{AOB}(\mathrm{S}))$ as a predictor for word learning in bilinguals.

\begin{tabular}{|l|l|l|l|}
\hline $\begin{array}{l}\text { Dependent } \\
\text { Variable }\end{array}$ & $\mathrm{AOB}(\mathrm{S})$ & SES & NWR \\
\hline Naming & $-.692^{* *}$ & $.578^{* *}$ & -.081 \\
\hline Identification & $-.502^{* *}$ & $.328^{*}$ & .039 \\
\hline AOB(S) & - & $-.850^{* *}$ & .258 \\
\hline SES & - & - & -.305 \\
\hline
\end{tabular}

Note $* * \mathrm{p}<.01, * \mathrm{p}<.05, \mathrm{AOB}(\mathrm{S})=$ Age of onset of bilingualism (speaking), SES = Socio Economic Status, NWR= Non Word Repetition 